\title{
ARTICLE OPEN \\ Contribution of point defects and nano-grains to thermal transport behaviours of oxide-based thermoelectrics
}

\author{
Guang-Kun Ren ${ }^{1}$, Jin-Le Lan ${ }^{2}$, Kyle J Ventura ${ }^{3}$, Xing Tan ${ }^{1}$, Yuan-Hua Lin ${ }^{1}$ and Ce-Wen Nan ${ }^{1}$
}

Point defects and nano-grains are very effective ways to control the thermal conductivity in oxide-based thermoelectrics. Here we use the optimised Debye-Callaway model to understand how the effect of point defects and nano-grains to reduce the thermal conductivity by inducing normal process and oxygen vacancy in oxide-based thermoelectrics. Our results reveal that this model can be effective to fit the experimental data of thermal conductivity in $\mathrm{ZnO}-, \mathrm{CaMnO}_{3}-, \mathrm{BiCuSeO}_{-}, \mathrm{SrTiO}_{3}-\mathrm{and}_{\mathrm{In}} \mathrm{O}_{3}$-based systems, which indicate that the normal scattering process and the oxygen vacancy will make obvious contribution to the thermal conductivity as compared with alloy compounds system. These calculations also propose that it could be desirable to obtain higher $Z T$ by controlling the concentration of oxygen vacancy in the nano-grained thermoelectric oxides.

npj Computational Materials (2016) 2, 16023; doi:10.1038/npjcompumats.2016.23; published online 12 August 2016

\section{INTRODUCTION}

On the basis of Seebeck and Peltier effect theory, thermoelectric (TE) materials and devices can directly convert heat to electricity or vice versa, without any moving mechanical parts, and are therefore silent and scalable. ${ }^{1}$ The conversion efficiency $(\eta)$ of TE materials is generally characterised by the dimensionless figure of merit: $Z T=S^{2} \sigma T /\left(\kappa_{\mathrm{L}+} \kappa_{\mathrm{e}}\right)$, where $S, \sigma, T, \kappa_{\mathrm{L}}$ and $\kappa_{\mathrm{e}}$ are the Seebeck coefficient, the electrical conductivity, the absolute temperature, the lattice thermal conductivity and the electronic thermal conductivity, respectively. ${ }^{2}$ To improve the $Z T$ of TE materials, attempts are often made to decrease the thermal conductivity $(\kappa)$ without detrimentally influencing the Seebeck coefficient and electrical conductivity. As of now, researchers have utilised two strategies to enhance TE performance. One is through improving power factor $\left(P F=S^{2} \sigma\right)$, based on band engineering, ${ }^{3,4}$ defect chemistry ${ }^{5,6}$ and quantum confinement effect, ${ }^{7-10}$ which was used for materials with a medium PF and a low thermal conductivity. Nevertheless, for systems with both relatively high thermal properties and tunable electrical performance, enlarging phonon scattering as the second strategy could be directly implemented to reduce intense heat transport. ${ }^{11-13}$

As for alloys, many researchers undertook a significant amount of theoretical explorations on reducing $K$ by phonon scattering, these materials including $\mathrm{CoSb}_{3}$ (refs 14-16), SiGe (ref. 17), hexagonal-CulnS 2 (ref. 18), half-Heusler alloys, ${ }^{19,20} \mathrm{Bi}_{2} \mathrm{Te}_{3}$ (refs 21,22) and LAST. ${ }^{23}$ The results indicated that phonon scattering of most TE alloys is induced by boundary scattering at low temperatures, by defect scattering at intermediate temperatures and by the Umklapp processes at high temperatures. Despite a number of methods successfully reducing $k$, they all came to the same conclusion that different patterns scatter different phonons of specific wavelengths at the optimal temperature for most related systems. For example, devices assembled with $\mathrm{Bi}_{2} \mathrm{Te}_{3}$ system are applied for low-temperature applications $(\sim 300 \mathrm{~K})$, hence strategies such as alloying with other elements and melt spinning to introduce plentiful nano-grains were developed to achieve better efficiency of grain or phase boundary scattering, ${ }^{24}$ further enhancing the comprehensive TE properties.

Compared with alloyed materials, TE oxides are nontoxic, widespread and inexpensive, moreover they are much more chemically and thermally stable. Considering that most TE oxides are suitable for regulating carrier concentration and electrical properties $\left(100-200 \mathrm{~S} \mathrm{~cm}^{-1}\right)$, and their Seebeck coefficients are comparatively high or approach coordinate level as in alloys, we focus more on strategies to decrease thermal conductivity. However, different from alloys including metallic or van der Waals bonds, strong covalent and ionic bonds exist in TE oxides lead to a relatively intrinsic high $\kappa\left(\sim 2-10 \mathrm{~W} \mathrm{~m}^{-1} \mathrm{~K}^{-1}\right)$. As a result, among all p-type TE oxides, $\mathrm{Ca}_{3} \mathrm{Co}_{4} \mathrm{O}_{9}$ has been intensively investigated and the highest $Z T$ value of 0.61 at $1,118 \mathrm{~K}$ was achieved by heavy doping and metallic nano-inclusion approach, ${ }^{25}$ which was still an inferior ZT. As for $n$-type oxides (e.g., $\mathrm{In}_{2} \mathrm{O}_{3}, \mathrm{SrTiO}_{3}, \mathrm{ZnO}$ and $\left.\mathrm{CaMnO}_{3}\right)$, the $Z T \mathrm{~s}$ are even lower.

Herein, we review recent progress of experiments and theoretical developments on reducing thermal properties of oxide-based TE materials and finally provide a clear proposal on how to organise proper point defects and sufficient nano-grains, for the sake of achieving corresponding lowest lattice thermal conductivities.

\section{RESULTS AND DISCUSSION}

Approach

To analyse regular thermal properties of most materials, relaxation time $\left(\tau_{c}\right)$ approximation of phonon gas is applied using Equation (1), which is provided by Debye. ${ }^{26} C_{V}$ represents the isochoric heat capacity, $I_{\text {ph }}$ the average phonon mean free path (MFP; $\left.I_{\text {ph }}=\tau_{c} \cdot v_{m}\right)$

\footnotetext{
${ }^{1}$ State Key Laboratory of New Ceramics and Fine Processing, Department of Materials Science and Engineering, Tsinghua University, Beijing, China; ${ }^{2}$ State Key Laboratory of Organic-inorganic Composite, College of Materials Science and Engineering, Beijing University of Chemical Technology, Beijing, China and ${ }^{3}$ School of Engineering, Clarkson University, Potsdam, NY, USA.

Correspondence: Y-H Lin (linyh@mail.tsinghua.edu.cn)

Received 27 January 2016; revised 26 June 2016; accepted 6 July 2016
} 
and the mean velocity of sound $\left(v_{m}\right)$ could be calculated by the combination of transverse branch $\left(v_{t}\right)$ and longitudinal branch $\left(v_{1}\right)$.

$$
\kappa_{L}=\frac{1}{3} C_{V} I_{p h} v_{m}
$$

For bulk materials, $v_{\mathrm{m}}$ can be directly described as $v_{m}=$ $\left(\frac{1}{3}\left(\frac{2}{v_{t}^{3}}+\frac{1}{v_{1}^{3}}\right)\right)^{-\frac{1}{3}}$ (ref. 27), further reduced to $v_{m}=\left(\frac{1}{2}\left(\frac{1}{v_{t}^{2}}+\frac{1}{v_{t}^{2}}\right)\right)^{-\frac{1}{2}}$ for two-dimensional thin film. ${ }^{28}$ When it comes to using first-principle calculations, the contribution of each phonon mode should be given by

$$
\kappa_{\lambda}=\frac{1}{L_{x} L_{y} L_{z}} \sum_{\lambda, \vec{q}} \tau(\vec{q}) C_{p h}\left(\omega_{\lambda}\right)\left[v_{\lambda}(\vec{q}) \cdot \vec{n}\right]^{2},
$$

all modes would be summed up to calculate $\kappa_{\mathrm{L}}$. Overall, a small $C_{V}$ and low $I_{\text {ph }}$ combined with inferior $v_{\mathrm{m}}$ would lead to an inferior $\kappa_{\mathrm{L}}$ in TE materials.

To analyse intrinsic factors that affect $\kappa_{\mathrm{L}}$, Slack's model ${ }^{29}$ as empirical formula should be mentioned first, which was given by

$$
\kappa_{L}=A \frac{\overline{\bar{M}} \Theta_{D}^{3} V^{1 / 3}}{\gamma^{2} n^{2 / 3} T},
$$

where $n$ is the number of atoms in the primitive cell, $V$ is the volume per atom, $\Theta_{\mathrm{D}}$ is the Debye temperature, $\bar{M}$ is the average atomic mass, $\gamma$ is the high-temperature limit of the acousticphonon Grüneisen parameter and $A$ represents a collection of physical constants that depends on $\gamma$. This model is accurate for simulating the lattice thermal conductivity of materials with relatively low $\Theta_{\mathrm{D}}$, such as $\mathrm{NaCl}, \mathrm{Nal}$ and $\mathrm{PbTe}$, but for materials with higher $\Theta_{D}(973 \mathrm{~K})$, the high-temperature Slack model cannot simulate experimental work well. ${ }^{30}$

To analyse $\kappa_{\mathrm{L}}$ for high temperatures, Eucken ${ }^{31}$ concluded on empirical grounds that many substances have the same $\kappa_{\mathrm{L}}$ at the melting point $T_{m}$, which appears to work well for materials with low melting points, but is futile for crystals melting above $500 \mathrm{~K}$. On the basis of this, Keyes proposed a better approximation at high temperatures: ${ }^{32}$

$$
\kappa_{L} T=\frac{R^{3 / 2} T_{m}^{3 / 2} \rho^{2 / 3}}{3 \gamma^{2} \varepsilon^{3} N_{0}^{1 / 3} \overline{\bar{M}}^{7 / 6}},
$$

where $\rho$ represents the density, $\varepsilon$ is the amplitude of thermal vibration of the atoms and $N_{0}$ is the Avogadro's number. Even this approximation provided no way of taking anisotropy into account, it identified $\kappa_{\mathrm{L}}$ of the covalently bonded solids tend to lie above that in the ionic and van der Waals solids, as well as introducing the effect of mass ratio, finally attributing the $\kappa_{\mathrm{L}}$ variation from material to material to variation of the average mass, the interatomic forces and the crystal structure.

On the basis of these models and relaxation time approximation, Debye-Callaway model divided the effect of phonon scattering into several parts, which can be written as:

$$
\kappa_{\mathrm{L}}=\frac{k_{B}}{2 \pi^{2} \nu}\left(\frac{k_{\mathrm{B}}}{h}\right)^{3} T^{3} \int_{0}^{\frac{\Theta_{D}}{T}} \frac{\tau_{\mathrm{C}} x^{4} e^{x}}{\left(e^{x}-1\right)^{2}} d x
$$

where $x=h \omega / k_{\mathrm{B}} T$ is a dimensionless quantity, $h$ Planck's constant, $k_{\mathrm{B}}$ the Boltzmann constant and $\omega$ the phonon frequency. The overall relaxation rate $\tau_{c}^{-1}$ in this model can be determined by combining the various scattering processes as given:

$$
\begin{aligned}
& \tau_{\mathrm{c}}^{-1}=\tau_{\mathrm{N}}^{-1}+\tau_{\mathrm{u}}^{-1}, \\
& \tau_{\mathrm{u}}^{-1}=\tau_{\mathrm{b}}^{-1}+\tau_{\mathrm{d}}^{-1}+\tau_{\mathrm{U}}^{-1}+\ldots,
\end{aligned}
$$

where, $\tau_{N}, \tau_{\mathrm{u}}, \tau_{\mathrm{b}}, \tau_{\mathrm{d}}$ and $\tau_{\mathrm{U}}$ are the relaxation times for normal process scattering, unnormal process scattering, grain boundary
(GB) scattering, point defect scattering and Umklapp scattering respectively, which are defined as:

$$
\begin{aligned}
& \tau_{\mathrm{N}}^{-1}=\delta \tau_{\mathrm{U}}^{-1}, \\
& \tau_{\mathrm{b}}^{-1} \propto v_{\mathrm{m}} / L, \\
& \tau_{\mathrm{d}}^{-1}=B \omega^{4}=\frac{V}{4 \pi v^{3}} \Gamma \omega^{4}, \\
& \tau_{\mathrm{U}}^{-1} \sim \omega^{a}\left(T / \Theta_{\mathrm{D}}\right)^{\beta} \exp \left(-\Theta_{\mathrm{D}} / b T\right),
\end{aligned}
$$

$\delta$ is a dimensionless constant, which describe relative intensity between normal and Umklapp process, $L$ is the grain size for polycrystalline sample, $\Gamma$ is the disorder scattering parameter and $B$ is a constant that is independent of temperature and frequency, represents mass and strain-stress fluctuations. There is no satisfactory theoretical work for the relaxation time model to be used in Umklapp and normal processes. However, Slack reported an expression for the relaxation time to be used in Umklapp model as given in Equation (11), in which $a, \beta$ and $b$ are constants. ${ }^{33}$ There should be many other processes included in these materials that affect the comprehensive thermal properties, such as electron-phonon interactions, dislocation and second-phase effects. Although considering their impacts are so small in most systems, for the sake of simplicity, most researchers choose to ignore these functions in the simulations.

As the momentum of the normal process remain conservative, the rate of change of the total phonon momentum due to normal process has to be zero, therefore this process does not give rise to thermal resistance. As a result, the original model ${ }^{14,20}$ used for most alloys ignores the normal process and only considers unnormal processes that do not conserve momentum. However, the normal process would affect the phonon concentration for whole range of frequencies and have the profound effect of transferring energy between different phonon modes.

\section{Point defects effect}

As researchers have found, the ultralow heat conduction of amorphous solids arises from phonons whose $I_{\mathrm{ph}}$ is comparable to the interatomic spacing. Introduced point defects are very useful in triggering these interatomic effects. ${ }^{19}$ Therefore, advantageous dopants include equivalent and aliovalent ion doping not only for the application of the modulation of electrical transport modulation and band engineering but they could also be of use for reducing thermal conductivity. Nevertheless, how to compare experimental work with theoretical analysis is a big challenge, as we know that different crystal structures, oxygen vacancy concentrations, various atomic masses and sizes should all have a significant impact.

Fortunately, these factors would reflect in their consequent effects. For example, whatever dopants they are, different atom sizes and masses trigger strain-stress and mass fluctuations. These fluctuations would enlarge the scattering of phonons with particular wavelengths, which is indicated by Debye-Callaway model, as given by Klemense from Equations (10 and 12, 13, 14):

$$
\Gamma=\Gamma_{M F}+\Gamma_{S F},
$$

$$
\begin{gathered}
\Gamma_{\mathrm{MF}}=\frac{\sum_{i=1}^{n} c_{i}\left(\frac{\overline{M_{i}}}{\overline{\bar{M}}}\right)^{2} f_{i}^{1} f_{i}^{2}\left(\frac{M_{i}^{1}-M_{i}^{2}}{\overline{M_{i}}}\right)^{2}}{\sum_{i=1}^{n} c_{i}} \\
\Gamma_{\mathrm{SF}}=\frac{\sum_{i=1}^{n} c_{i}\left(\frac{\overline{M_{i}}}{\overline{\bar{M}}}\right)^{2} f_{i}^{1} f_{i}^{2} \varepsilon_{i}\left(\frac{r_{i}^{1}-r_{i}^{2}}{\overline{r_{i}}}\right)^{2}}{\sum_{i=1}^{n} c_{i}},
\end{gathered}
$$

where the disorder scattering parameter $\Gamma$ is related to both strain field $\Gamma_{\mathrm{SF}}$ and mass fluctuation scattering $\Gamma_{\mathrm{MF}}, c_{i}$ is the relative 
degeneracy of the site, $f_{i}$ is the fractional occupation, $\overline{M_{i}}$ and $\bar{r}_{i}$ are the average mass and radii of that element, respectively, and $\bar{M}$ is the average atom mass. ${ }^{20}$ Therefore, by finding the impurity scattering coefficient $\Gamma$ through specific calculations and fitting, it is possible to predict a comprehensive $B$ and ultimately apply this value to analyse and further optimise the thermal conductivity of TE oxides.

Zinc oxide and its compounds become promising candidates for operation in high-temperature conditions. This is attributed to its excellent chemical and thermal stability at high temperatures, as well as superior electrical properties through elemental doping, nontoxic components and abundant natural resources. ${ }^{34}$ However, when phonons develop from the lattice vibrations and transport through the material, an orderly structure with insufficient defects results in a large mean phonon path $I_{\text {ph }}$ further restraining the intrinsic scattering mechanism. As we see in Figure 1a, different such as PGEC (phonon glass - electron crystal) materials characterised by very complex crystal structures and heavy atom compaction, the simple wurtzite structure of $\mathrm{ZnO}$ contains only two light elements and leads to less atom mass. Even if it has covalent properties-the electronegativities of $\mathrm{Zn}$ and $\mathrm{O}$ are 1.65 and 3.44, respectively-ionic bonds still dominate the whole material. Due to the strong interaction of ionic bonds and covalent bonds, phonons can transport between the crystal lattice with mostly harmonic vibration, and therefore the thermal conductivity of $\mathrm{ZnO}$ remains high during scattering processes with
$\kappa_{\mathrm{L}}$ over $40 \mathrm{~W} \mathrm{~m}^{-1} \mathrm{~K}^{-1}$ at room temperature, as Keyes mentioned. When the crystal structure changes from wurtzite binary $\mathrm{ZnO}$ to perovskite ternary compound $\mathrm{CaMnO}_{3}$, the $\mathrm{ABO}_{3}$ structure (Figure 1b) was more complex and multi-octahedral or tetrahedral intervals made spaces for other elements, even doping locations could increase to three, and the TE properties of $\mathrm{CaMnO}_{3}$ substituted at A and/or B sites have been widely investigatedthey have been found to depend on their preferable electrical performance. Furthermore, complex crystal structure made the intrinsic thermal conductivity of $\mathrm{CaMnO}_{3}$ reduce to only $3.6 \mathrm{~W} \mathrm{~m}^{-1} \mathrm{~K}^{-1}$ at room temperature when compared with $\mathrm{ZnO}$, and, similar to $\mathrm{ZnO}, \mathrm{CaMnO}_{3}$ has strong covalent or ionic bonds of $\mathrm{Mn}-\mathrm{O}, \mathrm{O}-\mathrm{O}$ and $\mathrm{Ca}-\mathrm{O}$; lack of weak linkages between atoms would limit the further decrease of $\kappa_{\mathrm{L}}$ value.

Figure 1c shows the tetragonal anti-PbO structure, which points to the layered oxides: $\mathrm{BiCuSeO}$. Its space group is $\mathrm{P} 4 / \mathrm{nmm}$, which includes $\left(\mathrm{Bi}_{2} \mathrm{O}_{2}\right)^{2+}$ and $\left(\mathrm{Cu}_{2} \mathrm{Se}_{2}\right)^{2-}$ layers. As the linkage between two layers is weak van der waals bonding, together with rather high Grüneisen constant $\gamma=1.5$, which indicates there should be contain inharmonic bonds that produce obstacles for phonon transport, ultimately heat cannot transport as fluidly as it does through oxides with strong bonds. Furthermore, theoretical heat capacity could be simply given by Dulong-Petit law:

$$
C=\frac{3 R}{\overline{\bar{M}}}
$$

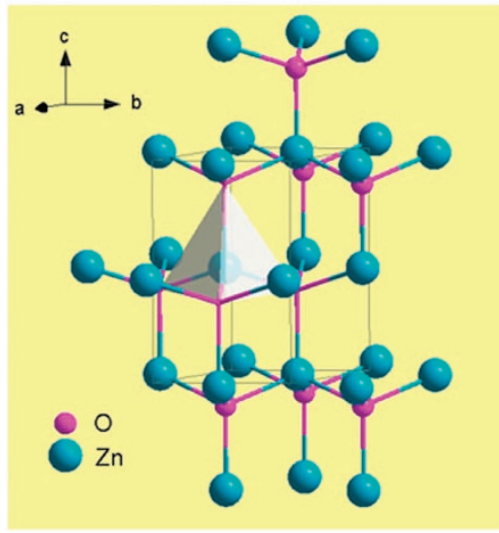

b

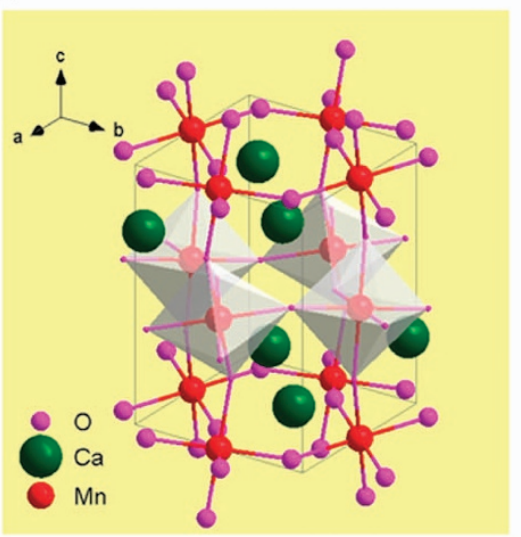

C

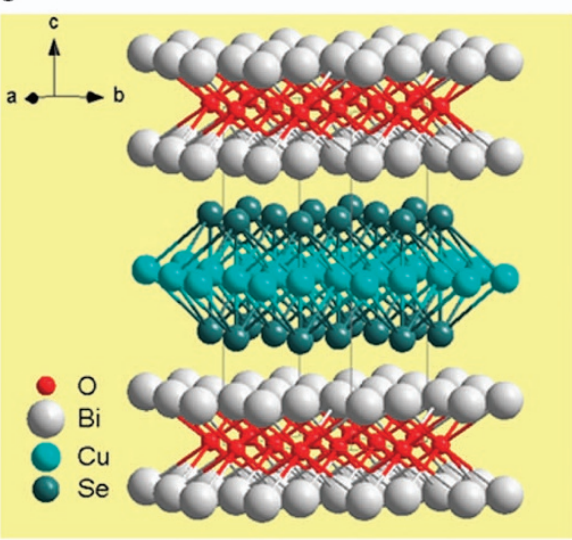

Figure 1. The crystal structure of (a) simple wurtzite oxide-based materials: $\mathrm{ZnO}\left(P 6_{3} m c\right)$, (b) perovskite oxides: $\mathrm{CaMnO}_{3}(P b n m)$ and $(\mathbf{c})$ layered oxides: BiCuSeO (P4/nmm).

a

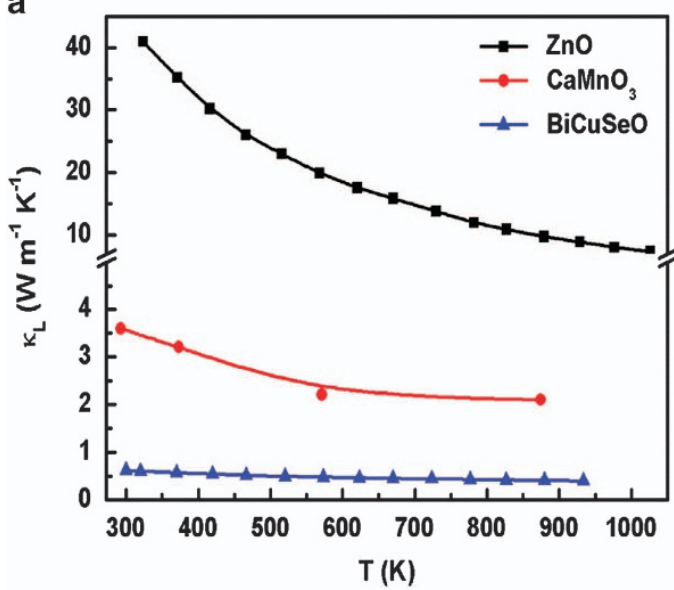

b

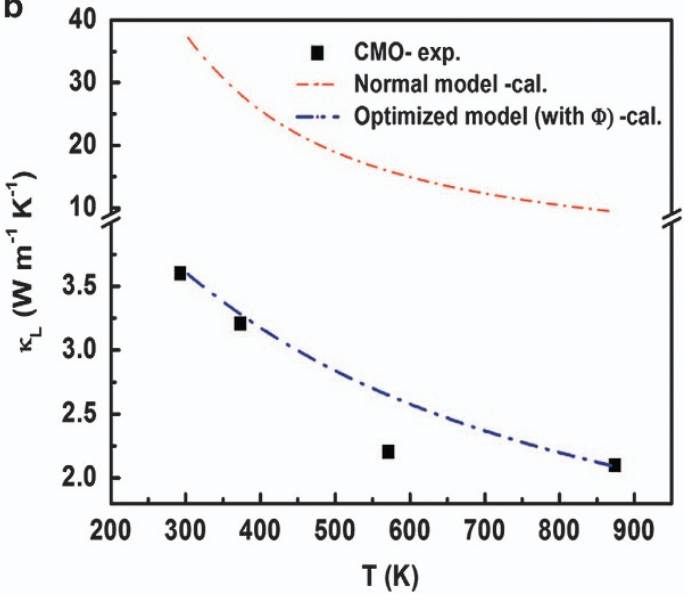

Figure 2. (a) Intrinsic $\kappa_{\mathrm{L}}$ of oxides with different crystal structures, (b) the comparison of calculated works through different models. 
when compared with the $\overline{\bar{M}}$ of $\mathrm{ZnO}\left(\overline{\bar{M}}_{\mathrm{ZnO}}=40.69\right)$ and $\mathrm{CaMnO}_{3}$ $\left(\overline{\bar{M}}_{\mathrm{CaMnO}_{3}}=28.60\right)$ the $\overline{\bar{M}}_{\mathrm{BiCuSeO}}$ was 91.86 and resulted in the lowest heat capacity among them $\left(C \sim 0.27 \mathrm{~J} \mathrm{~g}^{-1} \mathrm{~K}^{-1}\right)$. Combined all these special properties, the intrinsic $\kappa_{\mathrm{L}}$ was ultralow-only $0.65 \mathrm{~W} \mathrm{~m}^{-1} \mathrm{~K}^{-1}$ at room temperature, which was shown in Figure 2a.

To analyse and further organise efficient point defects for different kinds of oxides, we simulated some experimental work by relaxation time approximation. Therefore, we apply relaxation time approximation and Debye model in analysing the experimental results. However, different from alloys, the effect of
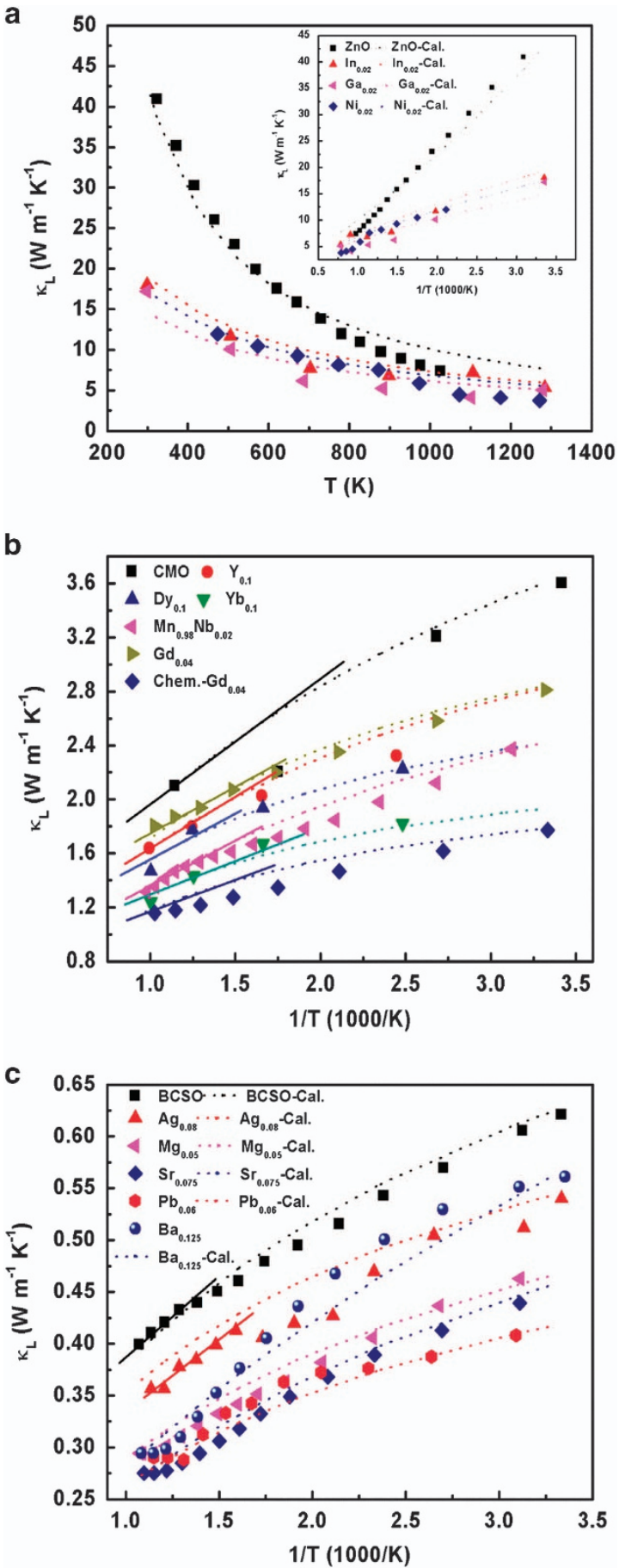

Figure 3. The thermal properties of (a) $\mathrm{ZnO}$, (b) $\mathrm{CaMnO}_{3}$ and (c) $\mathrm{BiCuSeO}$ with substitutional point defects. oxide vacancy should be considered. To prove this, first we took $\mathrm{CaMnO}_{3}$ as an example and compared the simulation work with and without oxygen vacancy part. For normal Debye model simulation work, we calculated the $\Gamma$ of the element doped into $\mathrm{CaMnO}_{3}$ was calculated by combining $\Gamma_{\mathrm{SF}}$ and $\Gamma_{\mathrm{MF}}$, which was found by taking the doping content, ionic size, atom mass and other physical parameters into Equations (13 and 14), and then getting $B$ for the point defect scattering process $\left(\tau_{d}\right)$ in the relaxation time approximation. Then, for optimised model, the simulation expression for $\tau_{d}^{-1}$ of oxides transfers from Equation (10) to vacancy-added formula, given by:

$$
\tau_{\mathrm{d}}^{-1}=B^{\prime} \omega^{4}=\frac{V}{4 \pi v^{3}}(\Gamma+\Phi) \omega^{4},
$$

where $B^{\prime}$ is the optimised constant, $\Phi$ is the scattering parameter for oxygen vacancy and it has an expression similar to $\Gamma$ in Equations $(12,13,14)$. Ultimately, with other unvarying $\tau_{N}, \tau_{b}$ and $\tau_{U}$, the simulation works are shown in Figure $2 b$ as dotted lines. The line fit the experimental work very closely for optimised Debye model, but for normal model the discrepancies between them are too large. This result led to the conclusion that existed oxygen vacancies should have an impact in scattering phonons for $\mathrm{CaMnO}_{3}$, as well as other oxides, which was distinct from alloys.

Seen from Figure $3 a$, the $\kappa_{\mathrm{L}}$ of pure $\mathrm{ZnO}$ fabricated by solid-state reaction (SSR) was reduced from $42 \mathrm{~W} \mathrm{~m}^{-1} \mathrm{~K}^{-1}$ at $300 \mathrm{~K}$ to $7.5 \mathrm{~W} \mathrm{~m}^{-1} \mathrm{~K}^{-1}$ at $1,023 \mathrm{~K}$, the inset graph exhibited $\mathrm{ZnO}$ following the $1 / \mathrm{T}$ relationship, which indicates phonon-phonon scattering charge for the tendency of high temperature. Once doped by other elements with the same coordination number such as $\mathrm{Ga}$, In, $\mathrm{Ni}$ or $\mathrm{Nb}$, the $\kappa_{\mathrm{L}}$ markedly decreased at room temperature and retained relatively small differences at high temperature. Listing some physical parameters in Table 1, and combined with simulated work through optimised mode, we consider the radius and mass of zinc as medium $\left(60 \mathrm{pm}, M_{\mathrm{Zn}}=65.41\right)$. Gallium (47 pm, $M_{\mathrm{Al}}=69.72$ ) with relatively small radius introduced large strain-stress fluctuations and indium ( $\left.62 \mathrm{pm}, M_{\mathrm{ln}}=114.82\right)$ with its relatively large mass introduced mass fluctuation, and these could be the reason for large loss in $\kappa_{\mathrm{L}}$, especially at room temperature.

Besides, the discrepancies between the simulations and experimental work come from the difference of theoretical and real densities, as well as radiation losses, the deviation of the thermal conductivity from the $1 / T$ relation, and temperature dependence of the Lorenz number. ${ }^{35}$

When it came to $\mathrm{CaMnO}_{3}$, though the undoped $\kappa_{\mathrm{L}}$ was much lower than $\mathrm{ZnO}$, as seen in Figure $2 \mathrm{a}$, after numerous dopants

\begin{tabular}{|lcccccc|}
\hline Table 1. & The fundamental physical parameters of doped elements \\
\hline Ion & Coordination & Charge & $\begin{array}{c}\text { Molar } \\
\text { mass } \\
(g)\end{array}$ & Electronegativity & $\begin{array}{c}\text { Crystal } \\
\text { radius } \\
(p m)\end{array}$ & $\begin{array}{c}\text { lonic } \\
\text { radius } \\
(p m)\end{array}$ \\
\hline $\mathrm{Zn}$ & 4 & 2 & 65.41 & 1.65 & 74 & 60 \\
$\mathrm{Ga}$ & 4 & 3 & 69.72 & 1.81 & 61 & 47 \\
$\mathrm{In}$ & 4 & 3 & 114.82 & 1.78 & 76 & 62 \\
$\mathrm{Ni}$ & 4 & 2 & 58.69 & 1.91 & 69 & 55 \\
$\mathrm{Ca}$ & 12 & 2 & 40.08 & 1.00 & 148 & 134 \\
$\mathrm{Dy}$ & 8 & 2 & 162.5 & 1.22 & 133 & 119 \\
$\mathrm{Yb}$ & 8 & 2 & 173.04 & 1.10 & 128 & 114 \\
$\mathrm{Gd}$ & 8 & 3 & 157.25 & 1.20 & 119 & 105 \\
$\mathrm{Y}$ & 8 & 3 & 88.94 & 1.22 & 116 & 102 \\
$\mathrm{Mn}$ & 6 & 4 & 54.94 & 1.55 & 67 & 53 \\
$\mathrm{Nb}$ & 6 & 3 & 92.91 & 1.60 & 86 & 72 \\
$\mathrm{Bi}$ & 8 & 3 & 208.98 & 2.02 & 131 & 117 \\
$\mathrm{Mg}$ & 8 & 2 & 24.31 & 1.31 & 103 & 89 \\
$\mathrm{Ba}$ & 10 & 2 & 137.33 & 0.89 & 166 & 152 \\
$\mathrm{Sr}$ & 8 & 2 & 87.62 & 0.95 & 140 & 126 \\
$\mathrm{~Pb}$ & 8 & 2 & 207.2 & 2.33 & 143 & 129 \\
$\mathrm{Ag}$ & 8 & 1 & 107.87 & 1.93 & 142 & 128 \\
\hline
\end{tabular}


were introduced into the lattice, we found elements such as Dy, $\mathrm{Yb}, \mathrm{Y}, \mathrm{Gd}$ and $\mathrm{Nb}$ (refs 36-41) would further reduced the $\kappa_{\mathrm{L}}$ significantly, as shown in Figure 3b. Furthermore, most of the efficient dopants are rare earth elements, including Dy, Yb and Gd, as seen in Table 1, attributed to higher mean atomic mass $(\bar{M})$, which leads to a relatively lower heat capacity $(C)$. We took classical expressions and optimised $\tau_{d}^{-1}$ in the simulation, and assumed that the normal process should have the same form as the Umklapp process (Equation (4)). Then, $\delta$ was set to be 4.3. Similar to $\mathrm{ZnO}$, the optimised Debye-Callaway model fitted the experimental $\kappa_{\mathrm{L}}$ of $\mathrm{CaMnO}_{3}$ very well, as seen from Figure $3 \mathrm{~b}$, whether it was undoped or doped.

From Figure $3 \mathrm{~b}$, these $\mathrm{CMO}$-based materials are in same fabrication but slightly different compositions. The $\kappa_{\mathrm{L}}$ of pure CMO synthesised by SSR was reduced from $3.6 \mathrm{~W} \mathrm{~m}^{-1} \mathrm{~K}^{-1}$ at $300 \mathrm{~K}$ to $2.1 \mathrm{~W} \mathrm{~m}^{-1} \mathrm{~K}^{-1}$ at $873 \mathrm{~K}$, showing that the $T^{-1}$ tendency at high temperature corresponds to phonon-phonon scattering. However, with some defects, the $\kappa_{\mathrm{L}}$ of $\mathrm{Ca}_{0.9} \mathrm{Y}_{0.1} \mathrm{MnO}_{3}$ markedly decreased from $1.82 \mathrm{~W} \mathrm{~m}^{-1} \mathrm{~K}^{-1}$ at $400 \mathrm{~K}$ to $1.23 \mathrm{~W} \mathrm{~m}^{-1} \mathrm{~K}^{-1}$ at $1,000 \mathrm{~K}$. Therefore, when compared with the pure sample, CMO with point defects in the Casite or the $\mathrm{Mn}$-site, as well as $\mathrm{O}$ vacancies, all reduced $\kappa_{\mathrm{L}}$ and could be regularly simulated. The theoretical scattering parameters were similarly obtained through above formulas, $\Theta_{D}$ values were estimated in this series of $C M O$ vary from 596.65 to $650 \mathrm{~K}^{42}$ Gruneision parameter ( $\gamma \sim 2.53-3.53$ ) was reported by Archana Srivastava et $a l^{43}$ and the mean velocity was $4,778 \mathrm{~m} \mathrm{~s}^{-1.42}$ After applying these parameters for calculations of Umklapp scattering, point defect scattering and boundary scattering processes, these terms finally gave rise to a comprehensive semi-theoretical thermal conductivity. Compared with experimental work, the oxygen vacancies were ubiquitous and make all the chemical formulae become $\mathrm{Ca}_{1-x} \mathrm{~A}_{x} \mathrm{Mn}_{1-y} \mathrm{~B}_{y} \mathrm{O}_{2.97}$. As $\mathrm{Yb}$ has the biggest mass difference $(173.04>40)$ and a considerable size difference $(102<134)$, it introduced the most phonon scattering among all the impurities. Therefore, little doping per cent would have a big role in reducing the lattice thermal conductivity of the samples, and the accuracy of these models also showed promising potential for predicting the impact of mass fluctuation and strain field effects of CMO.

As for BiCuSeO and its compounds, they were regarded as one kind of superconductors with the same anti-PbO structure as $\mathrm{LaBaCuO}$ in the beginning, then after no superconductivity was found, its TE properties began to attract many researchers' attention. ${ }^{44}$ Basically, due to its native superlattice properties from the layered $\left(\mathrm{Bi}_{2} \mathrm{O}_{2}\right)^{2+}-\left(\mathrm{Cu}_{2} \mathrm{Se}_{2}\right)^{2-}$ structure, as shown in Figure $3 \mathrm{c}$, $\mathrm{BiCuSeO}$ exhibited a relatively high Seebeck coefficient, adjustable electrical conductivity and a very low thermal conductivity, the highest $Z T$ for this material was found to be was 1.4 at $973 \mathrm{~K}^{45}$ Moreover, this special material generally has a thermal and chemical stability similar to oxides but thermal properties similar to alloys, therefore we call it a semi-oxide rather than a traditional one.

According to Cahill's formulation, the glassy limit for thermal conductivity $\kappa_{\min }$ was estimated using:

$$
\kappa_{\min }=\frac{1}{2}\left(\frac{\pi}{6}\right)^{1 / 3} k_{\mathrm{B}} v^{-2 / 3}\left(2 v_{\mathrm{t}}+v_{\mathrm{l}}\right)
$$

the calculated value of $\kappa_{\min }$ was nearly $0.59 \mathrm{~W} \mathrm{~m}^{-1} \mathrm{~K}^{-1}$, which even higher than that of the experimental work $\left(\kappa_{\mathrm{L}} \sim 0.40 \mathrm{~W} \mathrm{~m}^{-1} \mathrm{~K}^{-1}\right){ }^{46}$ Until now, most introduced point defects in this material aimed at improving the electrical properties, few give attention to the thermal performance. Therefore, it is important to demonstrate the origin of this ultralow $\kappa_{\mathrm{L}}$ and whether point defects could potentially have a role or not in such an unusual oxide.

The graph in Figure $3 c$ exhibits a significant amount of experimental work including $\mathrm{Mg}_{-}^{-}{ }^{47} \mathrm{Ba}^{-}{ }^{45} \mathrm{Sr}_{-}{ }^{48} \mathrm{~Pb}_{-}^{-}{ }^{49}$ and $\mathrm{Ag}$ (ref. 50)-doped BiCuSeO, as well as undoped material. Among these dopants, Sr- and Pb-doped samples both demonstrated the lower thermal conductivity than the others, even nearly close to $0.25 \mathrm{~W} \mathrm{~m}^{-1} \mathrm{~K}^{-1}$ at $973 \mathrm{~K}$. Others have attributed this phenomenon to the considerable point defects, small grain size and high texturation of the samples. As this kind of material was more likely to be anisotropic and probably included some soft modes because of the presence of $\left(\mathrm{Cu}_{2} \mathrm{Se}_{2}\right)^{2-}$ layer. We assumed normal processes would scatter more phonons than other oxides, and the simulation line of undoped $\mathrm{BiCuSeO}$ we did before ${ }^{51}$ also demonstrated there should be normal scattering process other than the effect of point defects, boundary and Umklapp scattering. As a result, we set $\delta$ to be $3-7$. However, we did not consider oxygen vacancies in that $\mathrm{BiCuSeO}$, as it was fabricated via self-propagating high-temperature synthesis in air. As for BiCuSeO synthesised by SSR, considering that it was synthesised in a vacuum, oxygen vacancies should absolutely exist, and this is also be indicated by Figure $3 \mathrm{c}$-the $\kappa_{\mathrm{L}}$ was much lower at room temperature. Moreover, as many published works theorised that the holes in the undoped samples came from $\mathrm{Cu}$ vacancies, we also inserted the effect of intrinsic $\mathrm{Cu}$ vacancy into the simulation work.

The simulation results were very close to the real $\kappa_{\mathrm{L}}$ and indicated that our assumption was applicable and reasonable. As can be seen in the graph of $K_{\mathrm{L}}$ versus $1 / T$ in Figure $3 c$, undoped and $\mathrm{Ag}$-doped BiCuSeO followed the $T^{-1}$ law, differing from other doped samples that exhibit a $T^{-a}(a>1)$ relation. This indicated numerous doped scattering mechanisms have changed, which varied from a simple mix of Umklapp scattering and normal scattering to multi-mechanism scattering, including the effect of second nano-phase and other mechanisms. Besides, the simulation work also demonstrated that there has already existed an ultralow $\kappa_{\mathrm{L}}$ in $\mathrm{BiCuSeO}$ - properly introduced point defects would further reduce it. Nevertheless, doping work for this material was more efficient at room temperature rather than it was at high temperature. As the highest $Z T$ of $\mathrm{BiCuSeO}$ was discovered at high temperatures ( 823-923 K), other stratagems were needed to decrease the lattice thermal conductivity.

According to Debye-Callaway normal model, $\tau_{d}$ for point effect scattering should be proportional to $1 / \omega^{4}$. This means that point defect scattering would not have a significant effect on low-frequency phonons, even diatomic doping could be simply interpreted as the overlay of two monatomic doping for the sake of enlarging doping limitation. Fortunately, plenty of research on alloy materials have proved that nanostructuring effects improve scattering for low-frequency phonons. In Figure 3b, Gd-doped CMO synthesised by a chemical coprecipitation method exhibited a much lower $\kappa_{\mathrm{L}}$ for the whole temperature range, even could be $\sim 1.1 \mathrm{~W} \mathrm{~m}^{-1} \mathrm{~K}^{-1}$ at $973 \mathrm{~K}$, which is ascribed to the higher concentration of grain boundaries caused by smaller grain sizes of 200-400 nm, compared with 3-5 $\mu \mathrm{m}$ particles fabricated by a SSR mechanism. ${ }^{41}$ Therefore, next we would introduce nano-grain effect and assumed it could also have an important role at high temperatures when the phonon-phonon scattering mechanisms dominated thermal transportation.

\section{Nano-grain effect}

Materials with nanostructure exhibit different properties from those of the corresponding bulk substances, single crystals, coarse-grained polycrystals and glasses, although they have an identical chemical composition. ${ }^{52}$ The two dominant concepts in the nanostructuring that affect the improvement of TE properties are quantum confinement by nano-scale constituents introduction and enhanced phonon scattering by the interfacial surfaces in nanostructures. The former contributes to high $S$ with relatively reduced $n$ by carrier filtering effect, whereas the latter reduces the $\kappa$ through nano-scale building blocks, which causes a significant increase in the density of grain boundaries. In the nanostructured 
bulk materials that have been tested, decrease of thermal conductivity has proven to be a primary mode of the enhancement in the TE figure of merit. ${ }^{34}$ Moreover, the size dependence of physical properties in nanostructured materials becomes evident when the size of building blocks is reduced to nanometre scale, which is comparable to the critical length of microphysical phenomena such as the MFPs of electrons or phonons. ${ }^{53}$ To select nano-scale as the target also considers the fact that the MFP of electrons and phonons have a big discrepancy at that length, phonons are efficiently scattered but electrons can successfully pass through. As a result, it reduced thermal conductivity and retained high carrier mobility simultaneously. ${ }^{54}$ As the MFP of low-frequency phonons is at a nanometre magnitude, materials fabricated with 1-100 nm grains would obviously introduce efficient boundary scattering. This phenomenon has been indicated by many early reports in TE alloys. $^{52}$

In the Debye-Callaway model, $\tau_{\mathrm{b}}^{-1}=\nu / L$ represents boundary scattering and it was closely connected to the microstructure of TE materials. As mean acoustic velocity, $v$ was one of the intrinsic properties that depended only on the composition itself, which was almost impossible to alter by varying the external environment. Nevertheless. $L$ generally could be taken for mean grain size, and negatively correlated to the whole relaxation time, further reducing the lattice contribution to heat transport. ${ }^{33}$

In this section, we report a study about TE oxides $\left(\mathrm{SrTiO}_{3}\right.$ and $\mathrm{In}_{2} \mathrm{O}_{3}$ ) with nanocrystals prepared by chemical methods or by a high-energy ball milling process. We then investigate the effect of nanocrystals on the $\kappa_{\mathrm{L}}$ of oxides, especially for high temperature, and analysed it through the optimised Debye-Callaway model, for the sake of giving an insight into the optimised grain size for lower thermal conductivity in oxides.

As shown in Figure 4a, similar to CMO, STO and its compounds are also a typical perovskite-type oxides, which have an isotropic cubic crystal structure $(a=3.904 \AA, P m \overline{3} m)$ above $105 \mathrm{~K}^{55}$ The strong structural tolerance makes substitutional doping in STO easier, and allows it to reach degenerate state and possess high electrical conductivity. ${ }^{56}$ Because of its high melting temperature of $2,353 \mathrm{~K}$, STO has the fundamental potential for application at very high temperatures, it can even be used as a middle transformation at nuclear electricity.

The conduction bands of STO are formed by Ti $3 d$ orbitals consisting of triply degenerate orbitals $\left(3 d_{x y}, 3 d_{y z}\right.$ and $\left.3 d_{x z}\right)$ and its valence bands formed by an $\mathrm{O} 2 p$ orbital, the bandgap $E_{\mathrm{g}}$ could be $3.2 \mathrm{eV}$. Therefore, STO simultaneously has large $S$ and relatively high modulated $n$, and the power factor of doped STO $\left(\sim 6.5 \mu \mathrm{W} \mathrm{cm}^{-1} \mathrm{~K}^{-2}\right)$ is promising enough to compete with conventional TE alloys. ${ }^{57}$ Nevertheless, considering the heat transport, lattice vibration contribution $\kappa_{\mathrm{L}}$ is almost one order of magnitude higher than those of conventional TE materials. Even with dopant cations as point defects, its relatively simple structure, limited doping level and lack of effective phonon scattering centres make it impossible to exhibit the effects of point defects. Therefore, the $Z T$ values of STO and its related compositions are still far below alloys ascribed to high thermal conductivity $\left(\sim 9.5-4.3 \mathrm{~W} \mathrm{~m}^{-1} \mathrm{~K}^{-1}\right)$ in the temperature range of $300-1,000 \mathrm{~K}$.

As shown in Figure $5 \mathrm{a}$, though the $\kappa_{\mathrm{L}}$ of $\mathrm{SrTiO}_{3}$ single crystal was relatively high, high concentration of GBs in STO polycrystalline ceramics, even micrometre grained, could scatter low-frequency phonons. Moreover, this result also indicated GB phonon scattering was much stronger in samples with smaller grains, though with increasing temperature the MFP of phonons were shortened and GB phonon scattering would be inevitably less significant at high temperatures. As a result, the lowest $\kappa_{\mathrm{L}}$ values observed in the $55 \mathrm{~nm}$-grained undoped STO was $5.3 \mathrm{~W} \mathrm{~m}^{-1} \mathrm{~K}^{-1}$ at $300 \mathrm{~K}$ and $3.4 \mathrm{~W} \mathrm{~m}^{-1} \mathrm{~K}^{-1}$ at $1,000 \mathrm{~K}$, corresponding to a $45 \%$ reduction and a $20 \%$ reduction, respectively, compared with $K_{\mathrm{L}}$ values of the bulk single crystal. Nevertheless, the reduction of $\kappa_{\mathrm{L}}$ at $1,000 \mathrm{~K}$ in the $55 \mathrm{~nm}$-grained ceramic was only $\sim 20 \%$ of that in single crystal. This indicated that even a grain size as small as $55 \mathrm{~nm}$ was not sufficient to reduce the $\kappa_{\mathrm{L}}$ of a polycrystalline STO ceramic to the glassy limit $\left(\sim 1 \mathrm{~W} \mathrm{~m}{ }^{-1} \mathrm{~K}^{-1}\right)$ because it was still far larger than the phonon MFP, which is given in Figure $5 b^{58}$

$$
I_{\mathrm{ph}}=20 T_{\mathrm{m}} d / \mathrm{r}^{2} T \text {, }
$$

with melting point $T_{\mathrm{m}}$, lattice parameter $d$, Gruneisen constant $\gamma$ (usually $1-2$ ) and absolute temperature $T$. In Figure $5 b, I_{\text {ph }}$ decreases from $25 \mathrm{~nm}$ at $300 \mathrm{~K}$ to $10 \mathrm{~nm}$ at $1000 \mathrm{~K}$; meanwhile, the critical grain size for satisfactory reduction in $K_{\mathrm{L}}$ was estimated to have a comparison with MFP, therefore to adjust it to be 10-25 nm would be much more appropriate.

To clarify this thermal transport behaviour, the effective $\kappa_{\mathrm{L}}$ values were plotted as a function of reciprocal temperature in the inset graph of Figure $5 \mathrm{a}$, and simulation work through the optimised Debye-Callaway model are shown in Figures $5 \mathrm{c}$ and $\mathrm{d}$. The physical parameters come from Sakhya et al. ${ }^{59}$ Figure $5 a$ demonstrated that the $\kappa_{\mathrm{L}}$ value for a STO single crystal has a strong linear dependency on $1,000 / T$ in the whole temperature range, which meant that characteristic phonon-phonon scattering, the Umklapp process, was predominant in it. However, even the simulation line of a single crystal calculated by the optimised Debye-Callaway model was good, as shown in graph a

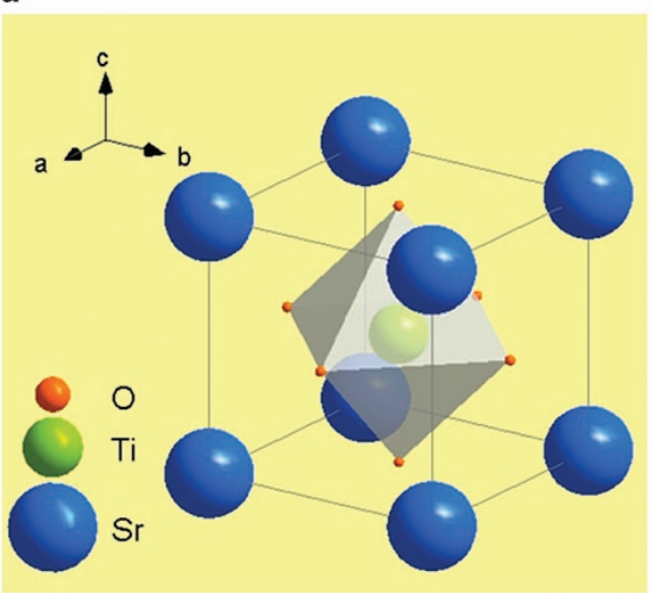

b

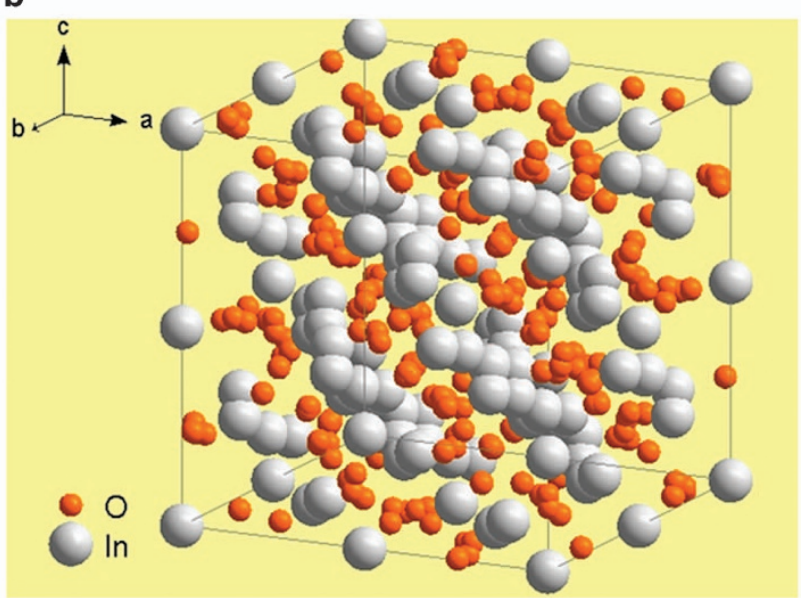

Figure 4. The crystal structure of (a) perovskite oxides: $\mathrm{SrTiO}_{3}(P m \overline{3} m)$ and (b) bixbyite oxides: $\ln _{2} \mathrm{O}_{3}(l a 3)$. 
a

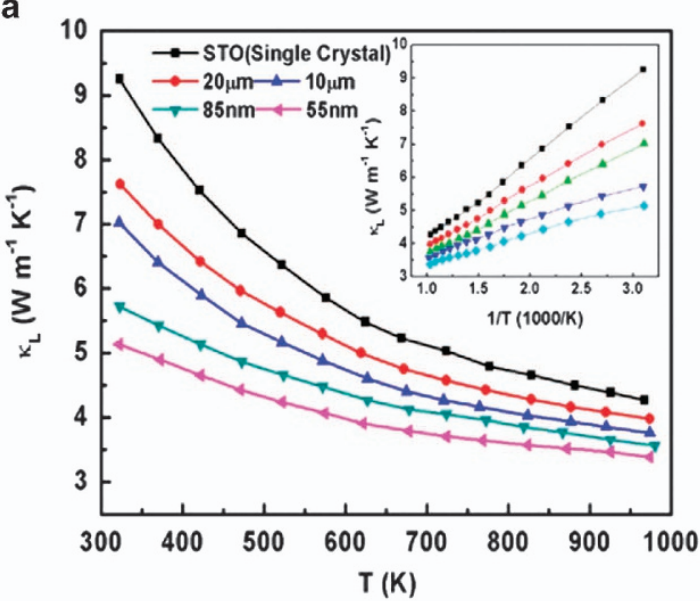

c

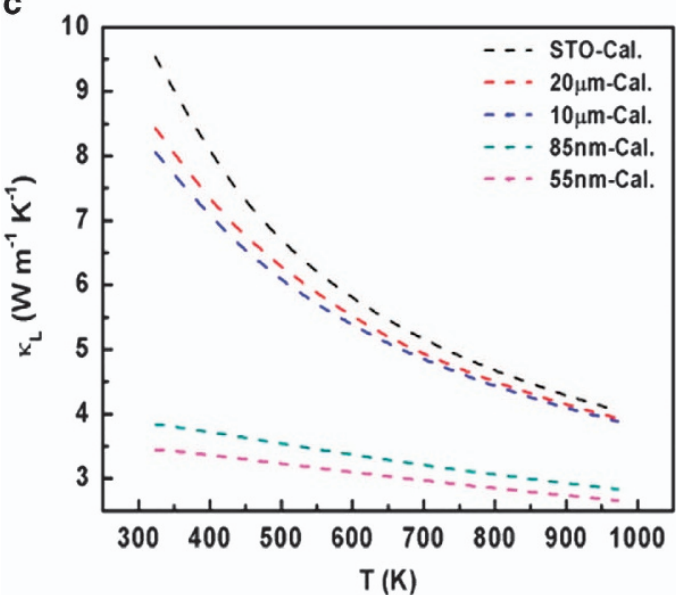

b

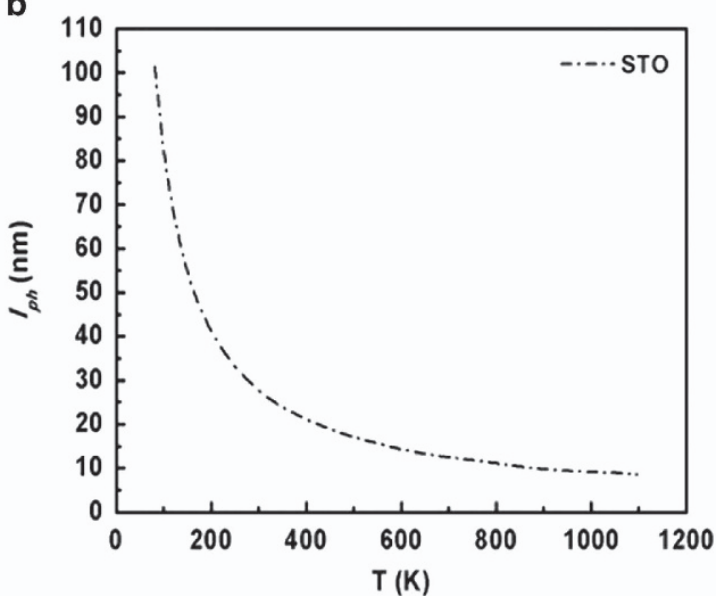

d

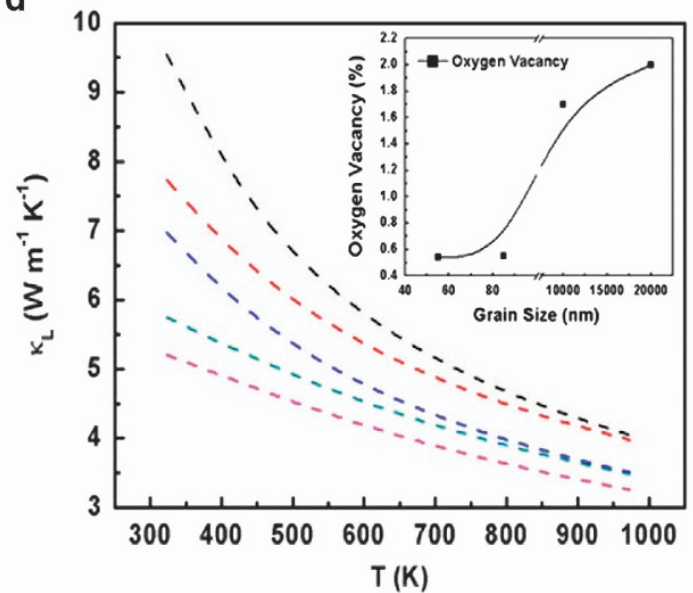

Figure 5. (a)Temperature dependence of lattice thermal conductivity: STO single crystal and polycrystalline ceramics with different grain sizes, the inset graph is a function of reciprocal temperature with $\kappa_{\mathrm{L}}{ }^{56}(\mathbf{b})$ temperature dependency of phonon mean free path for STO ceramics, (c) $\kappa_{\mathrm{L}}$ calculated by optimised Debye-Callaway model (the content of oxygen stays the same among all samples) and (d) $\kappa_{\mathrm{L}}$ calculated by optimised Debye-Callaway model (with variable oxygen vacancies which depends on grain size), the inset graph is grain size dependency of oxygen vacancy for STO ceramics.

Figure $5 c$, but when considering grain boundary scattering approximated by $\tau_{\mathrm{b}}^{-1}=\nu / L$, the discrepancies exhibited there should have some compensation in oxides, both for micrometrelevel and nanometre-level grain sizes.

We suspected the reason for the discrepancies could come from the content of oxygen vacancies and the fact that nano-grains were not easy to produce oxygen vacancies as micron-sized polycrystals. On the basis of this assumption, we repeated the simulation by considering various oxygen vacancy content for different grain size, as shown in graph Figure $5 d$ and its inset graph. The results corresponded with experimental work very well, which indicated the phenomenon that partial loss of oxygen vacancy in smaller grain size was highly possible at same synthesis condition. Therefore, to adjust the fabrication process for samples with different grain sizes was important, for the sake of retaining oxygen vacancies.

Besides, when combining the effect of point defects and nano-grain together, as shown in Figure $6 \mathrm{a}$, the $\kappa_{\mathrm{L}}$ of La-doped STO with $69 \mathrm{~nm}$ grain size exhibited regular tendency, more dopants led to lower $\kappa_{\mathrm{L}}$ at the basis of GB effect. Ultimately, the lowest $\kappa_{\mathrm{L}}$ was $2.3 \mathrm{~W} \mathrm{~m}^{-1} \mathrm{~K}^{-1}$ at $973 \mathrm{~K}$ in 9 at\% doped sample, decreased $46.5 \%$ when compared with undoped one. Therefore, with one or two decades of nanometre grain and doping work of properly heavy elements combined, $\sim 1 \mathrm{~W} \mathrm{~m}^{-1} \mathrm{~K}^{-1}$ would be possible as next strategy for STO in the future. This strategy also works for $\ln _{2} \mathrm{O}_{3}$ and its compounds.

By contrast with the fast grain growth of $\mathrm{ZnO}$ during synthesis, the grain size of $\ln _{2} \mathrm{O}_{3}$ and its system is much easier to control, ${ }^{61}$ the reason should come from its different crystal structure. As shown in Figure $4 \mathrm{~b}$, the lattice structure (Space group: la3) of $\mathrm{In}_{2} \mathrm{O}_{3}$ was much more complex than that in $\mathrm{ZnO}$. On the basis of a relatively high $\mathrm{PF}, \mathrm{In}_{2} \mathrm{O}_{3}$ has been developed as a promising $\mathrm{TE}$ oxide when the cubic bixbyite structure becomes disordered with various cations substituted for indium ions, and furthermore, co-substitution can significantly enhance the solubility of the dopant, which can simultaneously increase the electrical resistivity and decrease thermal conductivity. ${ }^{62}$ On this basis, Lan et al. ${ }^{61,63}$ introduced $\mathrm{Zn}$ and $\mathrm{Ce}$ co-doping that significantly reduced the thermal conductivity by fabricating these nano-grained $\mathrm{In}_{2} \mathrm{O}_{3}$-based bulks, and thus improving the TE performance.

Similar to STO, as shown in Figure $6 \mathrm{~b}$, the $T^{-1}$ trend indicated that the intrinsic Umklapp processes dominate the phonon transport. Moreover, the smaller the composite size is, the smaller the $\kappa_{\mathrm{L}}$, and even closer to the glassy limit $1.2 \mathrm{~W} \mathrm{~m}^{-1} \mathrm{~K}^{-1}$. Specifically, the $\kappa_{\mathrm{L}}$ of the $50 \mathrm{~nm}$-grained sample can be decreased to $7.9 \mathrm{~W} \mathrm{~m}^{-1} \mathrm{~K}^{-1}$ at room temperature, and was much lower compared with that of the $2 \mu \mathrm{m}$ grained one, which is 13.2 W m $\mathrm{W}^{-1} \mathrm{~K}^{-1}$. At high temperatures, the $\kappa_{\mathrm{L}}$ decreased from $2.7 \mathrm{~W} \mathrm{~m}^{-1} \mathrm{~K}^{-1}$ in $2 \mu \mathrm{m}$-grained sample to $1.5 \mathrm{~W} \mathrm{~m}^{-1} \mathrm{~K}^{-1}$ in 
a

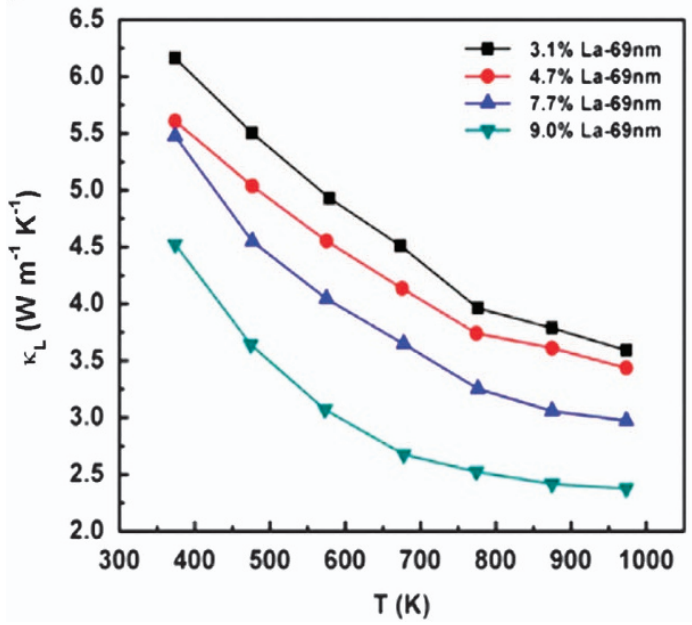

b

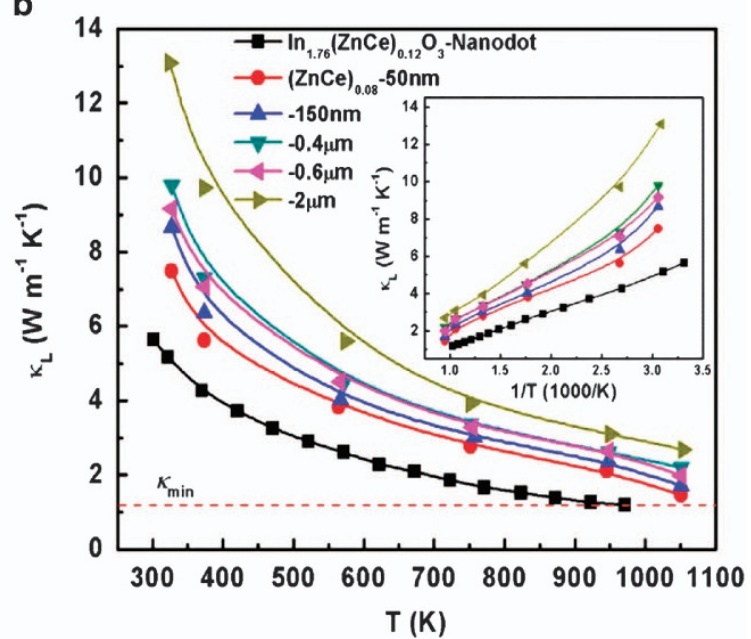

Figure 6. (a) Temperature dependence of effective thermal conductivity for La-doped STO with $69 \mathrm{~nm}$ grain size ${ }^{60}$ and (b) the lattice thermal property of $\ln _{2} \mathrm{O}_{3}$ with different grain sizes, the inset graph is the function with reciprocal temperature.

$50 \mathrm{~nm}$-grained samples, which clearly exhibited that the effect of nano-grains on phonon scattering also exists at high temperature.

Besides, based on this nano-grain work, when $\mathrm{CeO}_{2}$ came out as a second phase, nanodots are included in $\ln _{2} \mathrm{O}_{3}$. This took the lattice thermal conductivity even closer to the amorphous limit, and it was finally reduced down to $1.2 \mathrm{~W} \mathrm{~m}^{-1} \mathrm{~K}^{-1}$ at $973 \mathrm{~K}^{63}$ which is ascribed to the introduction of second-phase scattering:

$$
\tau_{\mathrm{sp}}{ }^{-1}=v\left({X_{\mathrm{s}}}^{-1}+\mathrm{X}_{\mathrm{sp}}{ }^{-1}\right)^{-1} V_{\mathrm{sp}},
$$

where $\chi_{s}$ represents the effects of intrinsic superficial area, $X_{s p}$ is the effect of introduced second phase and $V_{s p}$ is the number density of second phase.

'PGEC' should have complex structures with many elements, to produce scattering centres at a tiny scale. By contrast, $\ln _{2} \mathrm{O}_{3}$ as well as $\mathrm{ZnO}$ belongs to oxides with relatively simple structure and strong bonds between atoms, they do not satisfy this criterion, but our work shows that the effect of point defects and nanostructure in this material can be semi-quantified and estimated. Furthermore, they can be applied as efficient strategy and make simple oxides become potential candidates for TE materials.

\section{Conclusions}

Oxide-based TE materials have relatively high thermal conductivity, which limit their future applications. On the basis of the analysis of experimental work and the optimised Debye-Callaway model, we discovered point defects had a similar effect for both TE alloys and oxides. However, different from alloys, normal scattering process and oxygen vacancies $(\Phi)$ will make obvious contribution to the thermal conductivity for these oxide-based TEs by this model. On the basis of the calculated results, TE oxides could be promising candidates for future TE applications with nano-grain structures and optimum carrier concentration, as well as low thermal conductivity by appropriate point defects and suitable concentration of oxygen vacancies.

\section{ACKNOWLEDGEMENTS}

This work was financially supported by the National Key Research Programme of China, under grant no. 2016YFA0201003, the Ministry of Science and Technology of China through a 973-Project, under grant no. 2013CB632506, National Science Foundation of China under grant nos 51202232 and 11234012, and Specialized
Research Fund for the Doctoral Program of Higher Education, under grant no. 20120002110006.

\section{CONTRIBUTIONS}

G.-K.R. optimised the model for calculating thermal properties, and had primary writing responsibilities. J.L.L. carried out comparison with other theoretical models and made contribution to the writing process. K.J.V. and X.T. summarised experimental work and extensively edited the manuscript. Y.H.L. and C.W.N. organised the partnership and conceptualised, guided all aspects and led the project.

\section{COMPETING INTERESTS}

The authors declare no conflict of interest.

\section{REFERENCES}

1. Heremans, J. P. et al. Enhancement of thermoelectric efficiency in PbTe by distortion of the electronic density of states. Science 321, 554-557 (2008).

2. Snyder, G. J. \& Toberer, E. S. Complex thermoelectric materials. Nat. Mater. 7, 105-114 (2008).

3. Pei, Y., Wang, H. \& Snyder, G. Band engineering of thermoelectric materials. Adv. Mater. 24, 6125-6135 (2012).

4. Pei, Y. et al. Convergence of electronic bands for high performance bulk thermoelectrics. Nature 473, 66-69 (2011).

5. Rothschild, A., Menesklou, W., Tuller, H. L. \& Ivers-Tiffee, E. Electronic structure, defect chemistry, and transport properties of $\mathrm{SrTi}_{1-\mathrm{x}} \mathrm{Fe}_{\mathrm{x}} \mathrm{O}_{3-\mathrm{y}}$ solid solutions. Chem. Mater. 18, 3651-3659 (2006).

6. Porat, O. \& Riess, I. Defect chemistry of $\mathrm{Cu}_{2-\mathrm{y}} \mathrm{O}$ at elevated temperatures. Part II: electrical conductivity, thermoelectric power and charged point defects. Solid State lonics 81, 29-41 (1995).

7. Hicks, L. \& Dresselhaus, M. Effect of quantum-well structures on the thermoelectric figure of merit. Phys. Rev. B 47, 12727 (1993).

8. Hicks, L., Harman, T., Sun, X. \& Dresselhaus, M. Experimental study of the effect of quantum-well structures on the thermoelectric figure of merit. Phys. Rev. B 53, R10493 (1996).

9. Tian, Y. et al. One-dimensional quantum confinement effect modulated thermoelectric properties in InAs nanowires. Nano Lett. 12, 6492-6497 (2012).

10. Dresselhaus, M. S. et al. New directions for low-dimensional thermoelectric materials. Adv. Mater. 19, 1043-1053 (2007).

11. Hochbaum, A. I. et al. Enhanced thermoelectric performance of rough silicon nanowires. Nature 451, 163-167 (2008).

12. Kim, W. et al. Thermal conductivity reduction and thermoelectric figure of merit increase by embedding nanoparticles in crystalline semiconductors. Phys. Rev. Lett. 96, 045901 (2006).

13. Lehmann, T., Ryndyk, D. A. \& Cuniberti, G. Enhanced thermoelectric figure of merit in polycrystalline carbon nanostructures. Phys. Rev. B 92, 035418 (2015). 
14. Shi, X., Chen, L., Yang, J. \& Meisner, G. Enhanced thermoelectric figure of merit of $\mathrm{CoSb}_{3}$ via large-defect scattering. Appl. Phys. Lett. 84, 2301 (2004).

15. Nolas, G., Yang, J. \& Takizawa, H. Transport properties of germanium-filled $\mathrm{CoSb}_{3}$. Appl. Phys. Lett. 84, 5210 (2004).

16. Liu, W.-S., Zhang, B.-P., Zhao, L.-D. \& Li, J.-F. Improvement of thermoelectric performance of $\mathrm{CoSb}_{3-\mathrm{x}} \mathrm{Te}_{\mathrm{x}}$ skutterudite compounds by additional substitution of IVB-Group elements for Sb. Chem. Mater. 20, 7526-7531 (2008).

17. Cahill, D. G., Watanabe, F., Rockett, A. \& Vining, C. B. Thermal conductivity of epitaxial layers of dilute SiGe alloys. Phys. Rev. B 71, 235202 (2005).

18. Puzyrev, Y. S., Shen, X. \& Pantelides, S. T. Prediction of giant thermoelectric efficiency in crystals with interlaced nanostructure. Nano Lett. 16, 121-125 (2015).

19. Petersen, A., Bhattacharya, S., Tritt, T. M. \& Poon, S. J. Critical analysis of lattice thermal conductivity of half-Heusler alloys using variations of Callaway model. J. Appl. Phys. 117, 035706 (2015).

20. Yang, J., Meisner, G. \& Chen, L. Strain field fluctuation effects on lattice thermal conductivity of ZrNiSn-based thermoelectric compounds. Appl. Phys. Lett. 85, 1140 (2004).

21. Jeong, C., Datta, S. \& Lundstrom, M. Full dispersion versus Debye model evaluation of lattice thermal conductivity with a Landauer approach. J. Appl. Phys. 109, 073718 (2011)

22. Zhao, X. et al. Bismuth telluride nanotubes and the effects on the thermoelectric properties of nanotube-containing nanocomposites. Appl. Phys. Lett. 86, 062111 (2005)

23. Cook, B. A. et al. Analysis of nanostructuring in high figure-of-merit $\mathrm{Ag}_{1-\mathrm{x}} \mathrm{Pb}_{\mathrm{m}} \mathrm{SbTe}_{2+\mathrm{m}}$ thermoelectric materials. Adv. Func. Mater 19, 1254-1259 (2009).

24. Mun, H., Choi, S. M., Lee, K. H. \& Kim, S. W. Boundary engineering for the thermoelectric performance of bulk alloys based on bismuth telluride. ChemSusChem. 8, 2312-2326 (2015).

25. Van Nong, N., Pryds, N., Linderoth, S. \& Ohtaki, M. Enhancement of the thermoelectric performance of p-type layered oxide $\mathrm{Ca}_{3} \mathrm{Co}_{4} \mathrm{O}_{9+\delta}$ through heavy doping and metallic nanoinclusions. Adv. Mater. 23, 2484-2490 (2011).

26. Kittel, C. Introduction to Solid State Physics (Wiley, 2005).

27. Feng, J. et al. Calculation of the thermal conductivity of $\mathrm{L}_{2} \mathrm{SrAl}_{2} \mathrm{O}_{7}(\mathrm{~L}=\mathrm{La}, \mathrm{Nd}, \mathrm{Sm}$, Eu, Gd, Dy). Phys. Rev. B 84, 024302 (2011).

28. Lee, S. et al. Anisotropic in-plane thermal conductivity of black phosphorus nanoribbons at temperatures higher than 100 K. Nat. Commun. 6, 8573 (2015).

29. Morelli, D. T. \& Slack, G. A. In High Thermal Conductivity Materials 37-68 (Springer, 2006).

30. Zhang, Y., Dong, J., Kent, P. R., Yang, J. \& Chen, C. Intrinsic low thermal conductivity in weakly ionic rocksalt structures. Phys. Rev. B 92, 020301 (2015).

31. Eucken, A. Über die temperaturabhängigkeit der wärmeleitfähigkeit fester nichtmetalle. Ann. Phys. 339, 185-221 (1911).

32. Keyes, R. W. High-temperature thermal conductivity of insulating crystals: relationship to the melting point. Phys. Rev. 115, 564-567 (1959).

33. Yang, J. Low Thermal Conductivity Materials for Advanced Thermoelectric Applications. Dissertation for PhD. degree, University of Michigan, (2000).

34. Kinemuchi, Y. et al. Enhanced boundary-scattering of electrons and phonons in nanograined zinc oxide. J. Appl. Phys. 108, 053721 (2010).

35. Roufosse, M. C. \& Klemens, P. Lattice thermal conductivity of minerals at high temperatures. J. Geophys. Res. 79, 703-705 (1974).

36. Ohtaki, M., Koga, H., Tokunaga, T., Eguchi, K. \& Arai, H. Electrical transport properties and high-temperature thermoelectric performance of $\left(\mathrm{Ca}_{0.9} \mathrm{M}_{0.1}\right)$ $\mathrm{MnO}_{3}(\mathrm{M}=\mathrm{Y}, \mathrm{La}, \mathrm{Ce}, \mathrm{Sm}, \mathrm{In}, \mathrm{Sn}, \mathrm{Sb}, \mathrm{Pb}, \mathrm{Bi})$. J. Solid State Chem. 120 105-111 (1995).

37. Wang, Y. et al. High temperature thermoelectric response of electrondoped CaMnO3. Chem. Mater. 21, 4653-4660 (2009).

38. Bocher, L. et al. $\mathrm{CaMn}_{1-\mathrm{x}} \mathrm{Nb}_{\mathrm{x}} \mathrm{O}_{3}(\mathrm{x} \leq 0.08)$ perovskite-type phases as promising new high-temperature $\mathrm{n}$-type thermoelectric materials. Inorg. Chem. 47, 8077-8085 (2008).

39. Wang, Y., Sui, Y. \& Su, W. High temperature thermoelectric characteristics of $\mathrm{Ca}_{0.9} \mathrm{R}_{0.1} \mathrm{MnO}_{3}(\mathrm{R}=\mathrm{La}, \mathrm{Pr}, \ldots ., \mathrm{Yb})$. J. Appl. Phys. 104, 3703 (2008).

40. Zhang, F., Zhang, X., Lu, Q., Zhang, J. \& Liu, Y. Electronic structure and thermal properties of doped $\mathrm{CaMnO}_{3}$ systems. J. Alloy. Compd. 509, 4171-4175 (2011).
41. Lan, J. et al. High-temperature thermoelectric behaviors of fine-grained Gd-doped $\mathrm{CaMnO}_{3}$ ceramics. J. Am. Ceram. Soc. 93, 2121-2124 (2010).

42. Wang, Y., Sui, Y., Wang, X. \& Su, W. Enhancement of thermoelectric efficiency in (Ca, Dy) $\mathrm{MnO}_{3}-(\mathrm{Ca}, \mathrm{Yb}) \mathrm{MnO}_{3}$ solid solutions. Appl. Phys. Lett. 97, 052109 (2010).

43. Srivastava, A. \& Gaur, N. K. The role of lattice distortions in determining the thermal properties of electron doped $\mathrm{CaMnO}_{3}$ ). J. Phys. Condens. Matter 21, 096001 (2009).

44. Zhao, L.-D. et al. BiCuSeO oxyselenides: new promising thermoelectric materials. Energ. Environ. Sci. 7, 2900 (2014).

45. Sui, J. et al. Texturation boosts the thermoelectric performance of BiCuSeO oxyselenides. Energ. Environ. Sci. 6, 2916-2920 (2013).

46. Li, F., Wei, T.-R., Kang, F. \& Li, J.-F. Enhanced thermoelectric performance of Ca-doped $\mathrm{BiCuSeO}$ in a wide temperature range. J. Mater. Chem. A 1, 11942 (2013).

47. Li, J. et al. Thermoelectric properties of $\mathrm{Mg}$ doped p-type BiCuSeO oxyselenides. J. Alloy Compd. 551, 649-653 (2013).

48. Zhao, L. D. et al. $\mathrm{Bi}_{1-x} \mathrm{Sr}_{\mathrm{x}} \mathrm{CuSeO}$ oxyselenides as promising thermoelectric materials. Appl. Phys. Lett. 97, 092118 (2010).

49. Lan, J. L. et al. Enhanced thermoelectric properties of Pb-doped BiCuSeO ceramics. Adv. Mater. 25, 5086-5090 (2013).

50. Liu, Y.-C. et al. Influence of Ag doping on thermoelectric properties of BiCuSeO. J. Eur. Ceram. Soc. 35, 845-849 (2015).

51. Ren, G.-K., Butt, S., Ventura, K. J., Lin, Y.-H. \& Nan, C.-W. Enhanced thermoelectric properties in $\mathrm{Pb}$-doped $\mathrm{BiCuSeO}$ oxyselenides prepared by ultrafast synthesis. RSC Adv. 5, 69878-69885 (2015).

52. Toprak, M. S. et al. The impact of nanostructuring on the thermal conductivity of thermoelectric $\mathrm{CoSb}_{3}$. Adv. Func. Mater. 14, 1189-1196 (2004).

53. Gleiter, H. Nanostructured materials: basic concepts and microstructure. Acta Mater. 48, 1-29 (2000).

54. Rowe, D. \& Bhandari, C. Effect of grain size on the thermoelectric conversion efficiency of semiconductor alloys at high temperature. Appl. Energ. 6 347-351 (1980).

55. Lee, M. H. et al. Thermoelectric properties of $\mathrm{SrTiO}_{3}$ nano-particles dispersed indium selenide bulk composites. Appl. Phys. Lett. 102, 223901 (2013).

56. Koumoto, K., Wang, Y., Zhang, R., Kosuga, A. \& Funahashi, R. Oxide thermoelectric materials: a nanostructuring approach. Annu. Rev. Mater. Res. 40, 363-394 (2010).

57. Wang, N. et al. Enhanced thermoelectric performance of Nb-doped $\mathrm{SrTiO}$ by nano-inclusion with low thermal conductivity. Sc. Rep. 3, 3449 (2013).

58. Poirier, D. R. \& Geiger, G. H. Solutions Manual to Accompany Transport Phenomena in Materials Processing (John Wiley \& Sons Incorporated, 1994).

59. Sakhya, A. P. Electronic structure and elastic properties of $\mathrm{ATiO}_{3}(\mathrm{~A}=\mathrm{Ba}, \mathrm{Sr}, \mathrm{Ca})$ perovskites: a first principles study. Indian J. Pure Appl. Phy. 53, 102-109 (2015).

60. Park, K. et al. Colloidal synthesis and thermoelectric properties of La-doped $\mathrm{SrTiO}_{3}$ nanoparticles. J. Mater. Chem. A 2, 4217 (2014).

61. Lan, J. et al. High thermoelectric performance of nanostructured $\ln _{2} \mathrm{O}_{3}$-based ceramics. J. Am. Ceram. Soc. 95, 2465-2469 (2012).

62. Cheng, B. et al. Thermoelectric performance of $\mathrm{Zn}$ and GeCo-doped $\ln _{2} \mathrm{O}_{3}$ fine-grained ceramics by the spark plasma sintering. J. Am. Ceram. Soc. 94, 2279-2281 (2011).

63. Lan, J. L. et al. Enhanced thermoelectric performance of $\ln 203$-based ceramics via nanostructuring and point defect engineering. Sci. Rep. 5, 7783 (2015).

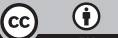

This work is licensed under a Creative Commons Attribution 4.0 nternational License. The images or other third party material in this article are included in the article's Creative Commons license, unless indicated otherwise in the credit line; if the material is not included under the Creative Commons license, users will need to obtain permission from the license holder to reproduce the material. To view a copy of this license, visit http://creativecommons.org/licenses/ by/4.0/

(c) The Author(s) 2016 